\title{
An Unusual Cause of Hemiplegia: External Hydrocephalus
}

\author{
Ralph Rahme, Ronald Moussa, Lina Menassa-Moussa, Elie Samaha
}

Can. J. Neurol. Sci. 2009; 36: 507-508

A well-known clinical entity in young children, external hydrocephalus $(\mathrm{EH})$ is a rare occurrence in adults that usually develops following head trauma or surgery for aneurysmal subarachnoid hemorrhage $(\mathrm{SAH})^{1-4}$. Neurological manifestations of EH in this population are generally similar to those of high or normal-pressure hydrocephalus ${ }^{1-4}$, although focal neurological deficits are occasionally reported ${ }^{3}$. In this paper, we present an unusual case of delayed post-operative EH presenting with acute hemiplegia after the resection of a recurrent meningioma.

\section{Case Report}

A 71-year-old male patient was referred to our service in July 2007 for a recurrent right pterional meningioma. The patient had been operated twice for this lesion, initially in 1996 and then in 2002, and gross total resection was achieved each time through a right pterional craniotomy. Pathological examination of surgical specimens revealed a meningothelial meningioma with low proliferation index (Ki-67 3-5\%) and no anaplastic features. A few months prior to his referral to us, the patient started developing progressive left-sided weakness and unsteady gait. Neurological examination showed mild left hemiparesis but was otherwise unremarkable. Magnetic resonance imaging (MRI) of the brain demonstrated recurrence of the right pterional meningioma extending posteriorly to the temporal horn of the right lateral ventricle, which appeared to be mildly dilated (Figures A, B). After discussing the different treatment options with the patient, the decision was made to surgically resect the lesion. Through a redo right pterional craniotomy, gross total resection of the tumor and its dural base was achieved extending into the right temporal horn, and duraplasty was performed using autologous pericranium. The patient had an uneventful postoperative course and was discharged home a few days later. At his one-month follow-up, his left hemiparesis and gait imbalance had completely resolved.

However, two months following his surgery, the patient woke up in the morning unable to move his left side and had urine all over his underwear. He was brought by his wife to the emergency room where examination revealed left hemiplegia, lethargy, and a tense subgaleal fluid collection overlying the craniotomy site. On further questioning, the patient's wife reported that the latter had been enlarging progressively over the past several days. At that time, our differential diagnosis for the patient's acute neurological event was stroke versus seizure plus postictal paralysis, although post-operative hydrocephalus was suspected given the growing subgaleal collection. An MRI of the brain was urgently performed and showed a right hemispheric subdural fluid collection communicating with the resection cavity and

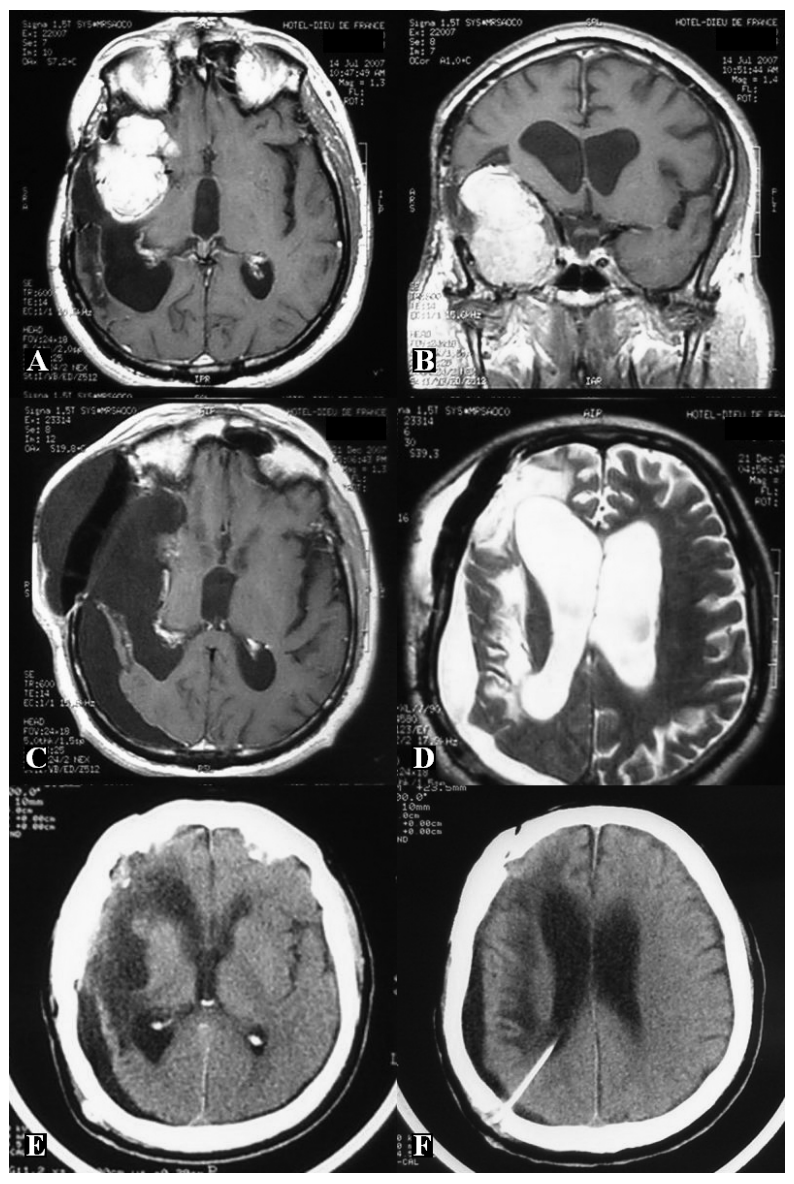

Figure: $A$ and $B$, axial $(A)$ and coronal $(B)$ contrast-enhanced T1WI brain MRI reveal a recurrent right pterional meningioma extending posteriorly to the temporal horn of the right lateral ventricle with a mildly dilated ventricular system. $C$ and $D$, axial contrast-enhanced T1WI (C) and unenhanced T2WI (D) brain MRI demonstrate gross total resection of the meningioma and a right hemispheric external hydrocephalus exerting significant mass effect on the adjacent cortex. E and F, Follow-up head CT one month after CSF diversion shows resolution of the external hydrocephalus and subgaleal collection.

From the Departments of Neurosurgery (RR, RM, ES), Radiology (LMM), SaintJoseph University and Hôtel-Dieu de France, Beirut, Lebanon.

Received February 3, 2009. Final Revisions Submitted March 18, 2009 Correspondence to: Ronald Moussa, Department of Neurosurgery, Hôtel-Dieu de France, Ashrafieh, Beirut, Lebanon. 
subgaleal collection and, through the right temporal horn, with the markedly dilated ventricular system. The collection appeared to exert a significant mass effect on the adjacent cortex with effacement of sulci (Figures C, D). In light of these findings, the diagnosis of $\mathrm{EH}$ was made and the patient was taken emergently to the operating room where a ventriculo-peritoneal shunt was inserted. Opening pressure of the lateral ventricle was extremely high. Post-operatively, the patient immediately regained full motor strength in his left side and a normal level of consciousness. His post-operative course was uneventful and he was discharged home a few days later. At his 1-month follow-up, he was completely asymptomatic, his subgaleal collection had resolved, and his neurological examination was unremarkable. Head computed tomogram demonstrated marked reduction in the size of the subdural collection and ventricular system (Figures E, F).

\section{Discussion}

External hydrocephalus is a well-defined clinical entity with a usually benign self-limited course in young children, where it is often associated with macrocephaly ${ }^{1}$. This condition has rarely been reported in adults, usually following head trauma or surgery for aneurysmal $\mathrm{SAH}^{1-4}$. Neurological manifestations of $\mathrm{EH}$ in adults are usually similar to those of high- or normal-pressure hydrocephalus and may include lethargy, altered mentation, cognitive dysfunction, headache, and gait imbalance ${ }^{1-4}$, although focal neurological deficits resulting from the mass effect exerted by the subdural collection are also occasionally reported ${ }^{3}$. In the present case, it is most likely that hemiplegia developed secondary to compression of the right cerebral hemisphere, particularly the primary motor cortex. This case is unusual for two reasons: first, $\mathrm{EH}$ developed following the resection of a recurrent meningioma in the absence of head trauma or SAH and second, acute hemiplegia was the patient's predominant symptom.

Although its pathophysiology is still unclear, EH is commonly believed to result from the combination of two phenomena:

- decreased absorption of cerebrospinal fluid (CSF) which may occur following SAH or trauma as a result of obstruction of CSF pathways and/or fibrosis of arachnoid granules, and

- tearing of the arachnoid membrane as a result of surgery or trauma, which may act as a ball-valve device leading to the subdural trapping of $\mathrm{CSF}^{2-4}$.
In some cases, epidural and subcutaneous fluid accumulation may occur as a result of further CSF egress through the dural sutures since excessive CSF tends to accumulate in the areas of least resistance within the intracranial cavity ${ }^{4}$. The patient described in this report might have developed reduced CSF absorption following his multiple craniotomies as a result of repetitive occult bleeding in the subarachnoid space and ventricles. This alteration in the patient's CSF hydrodynamics in addition to the surgically created tears in the arachnoid might have thus resulted in the development of external hydrocephalus.

Subdural hygroma (SDH) constitutes the main differential diagnosis of $\mathrm{EH}^{1-4}$. In contrast to $\mathrm{SDH}$, the lateral ventricles in patients with $\mathrm{EH}$ are usually increased in size, indicating disturbed CSF absorption ${ }^{1-4}$. It is extremely important to differentiate the two conditions since their treatment is quite different: while EH may be treated by inserting a CSF shunt, the latter may actually worsen a $\mathrm{SDH}^{1-4}$.

Although rare, post-operative EH may occur in adults in the absence of head trauma or subarachnoid hemorrhage and may present with focal neurological deficits. External hydrocephalus should be suspected whenever a subdural fluid collection is present along with other signs of disturbed CSF absorption such as an enlarged ventricular system and the presence of a subgaleal fluid collection. Differentiating EH from SDH is crucial since CSF shunting may treat the former but exacerbate the latter.

\section{REFERENCES}

1. Cardoso ER, Schubert R. External hydrocephalus in adults. Report of three cases. J Neurosurg. 1996; 85:1143-7.

2. Huh PW, Yoo DS, Cho KS, Park CK, Kang SG, Park YS, et al. Diagnostic method for differentiating external hydrocephalus from simple subdural hygroma. J Neurosurg. 2006; 105:65-70.

3. Kawaguchi T, Fujita S, Hosoda K, Shibata Y, Komatsu H, Tamaki $\mathrm{N}$. Treatment of subdural effusion with hydrocephalus after ruptured intracranial aneurysm clipping. Neurosurgery. 1998; 43:1033-9.

4. Yoshimoto Y, Wakai S, Hamano M. External hydrocephalus after aneurysm surgery: paradoxical response to ventricular shunting. J Neurosurg. 1998; 88:485-9. 